\title{
Anemia in Married Females of Uttar Pradesh and Its relation to Body Mass Index: Application of Poisson Regression
}

\author{
Brijesh P. Singh ${ }^{1}$, Sonam Maheshwari², Puneet Kumar Gupta ${ }^{3}$ \\ ${ }^{1}$ Faculty of Commerce \& DST-CIMS, Banaras Hindu University, Varanasi \\ ${ }^{2}$ Department of Community Medicine, SRMS-IMS, Bareilly \\ ${ }^{3}$ Department of Statistics, University of Allahabad, Allahabad
}

\begin{abstract}
Anemia is a common public health issue and multi-factorial condition which cuts across all the sections of the population and is associated with a variety of adverse outcomes, including mortality. According to the World Health Organization (WHO) anemia is defined as hemoglobin concentration in the blood. A female is anemic if hemoglobin concentration in the blood is less than $12 \mathrm{~g} / \mathrm{dl}$. Anemia is an indicator of poor nutrition and thus it is a public health issue which affects social and economic development of the region. The body mass index of married women is a high quality sign of a country's health status as well as economic condition and generally it has four categories i.e. underweight, normal weight, overweight and obese. Body Mass Index (BMI) provides an indicator for supporting to wipe out many preventable diseases. Alteration in nutritional status plays an important role in the course of a person's health. Hence, BMI can be used as an indicator for nutrition status, and association with some diseases can be expected. This study aimed to investigate the relationship between BMI and socioeconomic, demographic and health variables among 6723 currently married and non-pregnant women aged between 15-49 in Uttar Pradesh, India. In Indian population, overweight/obese women are significantly 86 percent more likely to be non-anemic, thus we may use BMI as a marker of anemia.
\end{abstract}

Key words: Body Mass Index (BMI), Poisson Regression, Anemia.

\section{Introduction}

Obesity poses one of the greatest public health challenges for the $21^{\text {st }}$ century in the developed world. The prevalence of obesity has increased in most countries over the last decade (WHO, 2003). Therefore, it is important to study its determinants. In this paper, we explore the empirical relationship between socioeconomic variables such as place of residence, religion, standard of living index, occupation and other demographic variables like respondent age, age at marriage and breast feeding. Also some health variables like anemia with body mass index using have been used. Poisson regression is used to find out the impact of the considered variable on BMI. In country like in India, women face serious health problems due to socioeconomic conditions, nutrition and rigid gender discrimination. A number of genetic and environmental factors governed the patterns of height growth from infancy to early adulthood but patterns of weight specially for women in India is mainly influenced by gender discrimination from birth (Miller 1983; Kishor 1998), inequitable distribution of health resources (Arnoldet et al. 1996; Basu 1995) and frequent reproductive cycling and infection (Koblinsky 1995; Brabinetal 1998; Bhatia \& Cleland 1995; Bang et al. 1989).

Obesity is associated with chronic inflammation (including elevated levels of hepcidin), thus obesity is associated with anemia of chronic disease (Cheng et al. 2011). Anemia is a global public health problem affecting both developing and developed countries with major consequences on human health as well as social and economic development. Anemia is the result of a wide variety of causes that can be isolated. Anemia among women causes many 
Regression

serious health problems and is pervasive in developing countries. The prevalence of anemia for ever-married women has increased from 52 percent in NFHS-2 to 56 percent in NFHS-III (for all sample data). Therefore, the anemia situation has worsened over time for women. Reason for high prevalence of anemia in these women could be due to age, socioeconomic condition, low food intake, increase tendency to lose weight for zero size figure combined with menstrual loses. Overall, 49.2 percent of the females are anemic found in the study sample. The prevalence of underweight, overweight and obesity are 29.9, 13.8 and 7.9 percent respectively. Also Findings reveal that the anemia and BMI is not positively associated and significant. The obese group of female has the highest concentration of hemoglobin $(12.15 \mathrm{~g} / \mathrm{dl})$ compared with other group of BMI.

Obesity is also related to socioeconomic status of the female. The seminal review of 144 studies by Sobal and Stunkard (1989) conducted 15 years ago demonstrated a direct relationship between socioeconomic status and obesity in developing societies. That is, men, women and children of higher socioeconomic status had a higher likelihood of being obese than those of lower socioeconomic status. There are also two very recent papers that focus on the role of income as a BMI. Cawley et al. (2008) and Schmeiser (2008) look at the impact of income on weight in the US. While Cawley et al. (2008) find no statistically significant effects, Schmeiser (2008) find that family income significantly raises the BMI. Cawley (2016) observed the impact of health insurance on preventive care and health behaviors. In this study we aimed to find out the impact of socioeconomic and demographic variables on body mass index in Uttar Pradesh, India.

\section{Data and Methodology}

For the present study, we use the data of NFHS-III. It provides nationally representative data on female's height, weight and hemoglobin level. Because anemia is such a serious health problem in India, NFHS-III undertook direct measurement of the hemoglobin levels women age 15-49. Measurements were taken in the field using the HemoCueHb 201+ analyzer. In less than one minute, the hemoglobin concentration is indicated on a digital read-out. In addition, height and body weight were measured by health coordinators, who had some medical background, were employed by IIPS for the supervision of data collection for biomarkers. The height and weight measurements in NFHS-III are used to calculate the BMI. The BMI is defined as weight in kilograms divided by height in meters squared $\left(\mathrm{kg} / \mathrm{m}^{2}\right)$. Whole sample is divided in to four categories according to body mass index the obese (BMI $\geq 30.00 \mathrm{~kg} / \mathrm{m}^{2}$ ), overweight (BMI $25.00-30.00 \mathrm{~kg} / \mathrm{m}^{2}$ ), normal weight (BMI $\left.18.50-25.00 \mathrm{~kg} / \mathrm{m}^{2}\right)$, underweight $\left(B M I<18.50 \mathrm{~kg} / \mathrm{m}^{2}\right.$ ) according to the standard mentioned in NFHS report. There are dependent variable is BMI which can be defined in category wise and independent variables are place of residence, religion, standard of living index, age of the respondent, anemia age at first marriage, respondent education. Variables were presented as percentage or means/standard deviations by BMI categories, with chi square test for categorical variables and ANOVA for continuous variables.

Poisson regression was used to estimate the association as prevalence rates (PRs) between BMI categories and anemia controlling for confounders including age, residence, socioeconomic status, education level and age of respondent and age of respondent at time of first marriage as well as occupation. When the response variable is in the form of a count we face a yet different constraint. Counts are all positive integers and for rare events the Poisson distribution is more appropriate since the Poisson mean is more than zero. In a study the generalized Poisson regression (GPR) model is considered for identifying the relationship between the number of accidents and some covariates (Felix et al. 2004) bu here in this study the simple poisson regression has been used for simplicity. The logarithm of the response variable is linked to a linear function of explanatory variables. The regression equation may be written as 
$\log (Y)=\beta_{0}+\beta_{1} X_{1}+\beta_{2} X_{2}+\ldots \ldots \ldots \ldots$

$Y=e^{\beta_{0}} \cdot e^{\beta_{1}} \cdot e^{\beta_{2}}$

Where, $\beta_{0}, \beta_{1}, \beta_{2}$ are the regression coefficients.

\section{Results and Discussion}

Table 1 presents general characteristics of the study population by all BMI categories. Here we observed the percentage of being overweight is increasing with increment in age up to age 40 , after this percentage is decreasing gradually. Age is statistically associated with BMI. In Urban area 64.3 and 83.2 percent women are overweight and obese respectively. It is also observed percentage of overweight and obese are drastically increasing according to standard of living index but it is also observed that percentage of underweight is high in the low standard of living index females. In Hindu females the trend is decreasing however in Muslims it is increasing. Higher education plays a vital role in the BMI as education is increasing the BMI is also increasing. The adverse pattern have been observed for the respondents occupation those who are not working they have high BMI. Female whose partners are in good jobs they have high BMI. Those who are watching TV they are either overweight or obese and it is obvious an because of the consumption of snack food lack of physical activity while women who watching TV, did not work were significantly more likely to be overweight or obese than their counterparts who were working outside of the home. Currently breastfeeding females are less likely to be overweight or obese. There is an increasing BMI pattern has been observed for female with high age at marriage.

From the table 2 we have estimates of poisson regression for various background characteristics. As age is increasing the chance of being higher BMI is significantly more. Urban women are 86 percent more likely to be overweight as compared to rural women. Regional variables account for many differences including market access, population density and climate. These variables can also capture dietary habits or practices, dietary references, price of meals, and opportunity cost of time or other preferences that are specific to some areas. These are main reasons behind the variation in probability of being overweight. It is also observed in table 2 that the women whose SLI is medium and high 92 percent and 2.5 times more prone to be high category of BMI respectively. Religion play a significant cultural factor because Non-Hindu women were more likely to be overweight or obese than women from Hindu women due to their fooding habit, in Muslims the consumption of non-vegetarian food is frequent. Education plays a significant indirect role in the gaining higher weight through the high socioeconomic status which may responsible for high BMI. Thus there is positive association between respondent education and BMI; the reasons behind this association that as education level is increases with age, this tables also reveals that BMI has tendency to be high with age and the second reason that women with high educated profile are missed out of physical work like agriculture, house work etc. Women working in professional, technical, managerial, office, clerical or sales positions were not significantly different in their probability of becoming overweight or obese compared with housewives but service class women are more likely to be overweight or obese, the risk is 72 percent more.. The probability of becoming overweight is significantly high in non-anemic women because of their good health condition due to high hemoglobin level. 
Regression

\section{Conclusion}

From the present study, we reveal that the high prevalence of anemia among women in Uttar Pradesh the most populous state of India is a burden for them, for their families, and for the economic development and productivity of the country. Iron supplementation programs, for a variety of reasons, have not been effective in reducing prevalence of anemia (Galloway \& McGuire 1994; Vijayaraghaven et al.1990). Poisson regression analysis shows that the prevalence rate of high BMI for non anemic women is 86 percent more. We suspect other problems may include acute infections, micronutrient deficiency that interferes with iron metabolism, or poor dietary patterns that compromise adequate iron intake. Among the urban, overweight and higher-income women with moderate to severe anemia, we suspect that diet may play an important role but we are unable to assess its role with the current data. From the above findings we may say BMI is a good marker of anemia. 
Brijesh P. Singh ${ }^{1}$, Sonam Maheshwari², Puneet Kumar Gupta ${ }^{3} 271$

Table1: Percent distribution of the Study Sample $(\mathrm{N}=6723)$ by BMI categories

\begin{tabular}{|c|c|c|c|c|c|}
\hline \multirow[b]{2}{*}{$\begin{array}{c}\text { Background } \\
\text { characteristics }\end{array}$} & \multicolumn{4}{|c|}{ Various categories of BMI } & \multirow{2}{*}{$\begin{array}{l}\text { p value } \\
\text { of the } \\
\text { test** }\end{array}$} \\
\hline & $\begin{array}{l}\text { Under } \\
\text { weight }\end{array}$ & $\begin{array}{c}\text { Normal } \\
\text { weight }\end{array}$ & $\begin{array}{c}\text { Over } \\
\text { weight }\end{array}$ & Obese & \\
\hline \multicolumn{6}{|l|}{ Age } \\
\hline $15-19$ & $128(6.4)$ & $201(6.2)$ & $10(0.9)$ & $3(0.7)$ & \multirow{7}{*}{0.000} \\
\hline $20-24$ & $362(18.0)$ & $571(17.5)$ & $90(8.8)$ & $4(0.9)$ & \\
\hline $25-29$ & $390(19.4)$ & $648(19.9)$ & $164(16.0)$ & $56(13.2)$ & \\
\hline $30-34$ & $416(20.7)$ & $628(19.3)$ & 201 (19.6) & $68(16.0)$ & \\
\hline $35-39$ & $328(16.3)$ & $563(17.3)$ & $237(23.1)$ & $86(20.2)$ & \\
\hline $40-44$ & $243(12.1)$ & $400(12.3)$ & $192(18.7)$ & $102(24)$ & \\
\hline $45-49$ & $141(7.0)$ & $248(7.6)$ & $132(12.9)$ & $106(24.9)$ & \\
\hline \multicolumn{6}{|l|}{ Residence } \\
\hline Urban & $490(24.4)$ & $1135(34.8)$ & $595(64.3)$ & $442(83.2)$ & \multirow{2}{*}{0.000} \\
\hline Rural & $1518(75.6)$ & $2124(65.2)$ & $330(35.7)$ & $89(16.8)$ & \\
\hline \multicolumn{6}{|l|}{ Standard of living Index } \\
\hline Low & 634 (31.6) & $637(19.5)$ & $52(5.6)$ & $15(2.8)$ & \multirow{3}{*}{0.000} \\
\hline Medium & $888(44.2)$ & $1224(37.6)$ & $185(20.0)$ & $36(6.8)$ & \\
\hline High & $486(24.2)$ & $1398(42.9)$ & $688(74.4)$ & $480(90.4)$ & \\
\hline \multicolumn{6}{|l|}{ Religion } \\
\hline Hindu & $1663(82.8)$ & $2670(81.9)$ & $720(77.8)$ & $408(76.8)$ & \multirow{3}{*}{0.000} \\
\hline Muslim & $341(17.0)$ & $560(17.2)$ & $180(19.5)$ & $106(20.0)$ & \\
\hline Others & $5(0.2)$ & $28(0.9)$ & $25(2.7)$ & $17(3.2)$ & \\
\hline \multicolumn{6}{|l|}{ Respondent's Education } \\
\hline Illiterate & $1420(70.7)$ & $1929(59.2)$ & $363(39.2)$ & $128(24.1)$ & \multirow{4}{*}{0.000} \\
\hline Primary & $203(10.1)$ & $387(11.9)$ & $111(12.0)$ & $57(10.7)$ & \\
\hline Secondary & $336(16.7)$ & $711(21.8)$ & $272(29.4)$ & $194(36.5)$ & \\
\hline Higher & $49(2.4)$ & $232(7.1)$ & $179(19.4)$ & $152(28.6)$ & \\
\hline \multicolumn{6}{|l|}{ Partner's Education } \\
\hline Illiterate & $722(35.9)$ & $844(25.9)$ & $129(13.8)$ & $53(9.6)$ & \multirow{4}{*}{0.000} \\
\hline Primary & $281(14.0)$ & $394(12.1)$ & $77(8.2)$ & $27(4.9)$ & \\
\hline Secondary & 864 (43.1) & $1555(47.8)$ & $442(47.9)$ & $261(49.3)$ & \\
\hline Higher & $141(7.0)$ & $464(14.3)$ & $277(30.0)$ & $191(36.1)$ & \\
\hline \multicolumn{6}{|l|}{ Respondent's Occupation } \\
\hline Professional/managerial & $8(0.4)$ & $48(1.5)$ & $42(4.5)$ & $20(3.8)$ & \multirow{5}{*}{0.000} \\
\hline Office/clerical/sales & $61(3.0)$ & $117(3.6)$ & $44(4.8)$ & $30(5.6)$ & \\
\hline Agriculture & $617(30.7)$ & $789(24.2)$ & $85(9.2)$ & $14(2.6)$ & \\
\hline Skilled/unskilled manual & $217(6.3)$ & 207 (6.4) & $56(6.1)$ & $24(4.5)$ & \\
\hline Not working & $1195(59.5)$ & $2098(64.4)$ & $698(75.5)$ & $443(83.4)$ & \\
\hline \multicolumn{6}{|l|}{ Partner's Occupation } \\
\hline Professional/managerial & $47(2.3)$ & $182(5.6)$ & $114(12.3)$ & $71(13.4)$ & \multirow{5}{*}{0.000} \\
\hline Office/clerical/sales & $391(19.5)$ & $783(24.1)$ & $348(37.8)$ & $248(46.8)$ & \\
\hline Agriculture & $700(34.9)$ & $931(28.6)$ & $151(16.4)$ & $48(9.1)$ & \\
\hline Skilled/unskilled manual & $845(42.1)$ & $1303(40.0)$ & $295(32.0)$ & $158(29.8)$ & \\
\hline Not working & $26(1.2)$ & $59(1.8)$ & $16(1.5)$ & $7(0.9)$ & \\
\hline \multicolumn{6}{|l|}{ Watching TV } \\
\hline Yes & $893(44.5)$ & $1940(59.5)$ & $740(80.0)$ & $487(91.7)$ & \multirow{2}{*}{0.000} \\
\hline No & $115(55.5)$ & $1319(40.5)$ & $185(20.0)$ & $44(8.3)$ & \\
\hline Currently Breast feeding & & & & & \\
\hline Yes & $846(42.1)$ & 1137 (34.9) & $146(15.8)$ & $61(11.5)$ & \\
\hline No & $1162(57.9)$ & $2122(65.1)$ & $779(84.2)$ & $470(88.5)$ & 0.000 \\
\hline Hemoglobin $(\mathrm{g} / \mathrm{dl})^{*}$ & $11.4(1.8)$ & $11.7(1.8)$ & $12.1(1.5)$ & $12.5(1.6)$ & 0.000 \\
\hline Age at first marriage* & $16.4(2.5)$ & $17.0(2.8)$ & $18.0(3.3)$ & $18.8(3.6)$ & 0.000 \\
\hline
\end{tabular}

*Mean (SD), **p value of chi square test for categorical variables and ANOVA for continues variables. 
Table 2: Incidence risk ratios of body mass index (BMI) among married women of Uttar Pradesh, India, Poisson regression analysis

\begin{tabular}{|c|c|c|c|c|}
\hline Background characteristics & $\mathbf{N}$ & IRR & $95 \% \mathrm{CI}$ & p value \\
\hline \multicolumn{5}{|l|}{ Age } \\
\hline $15-19$ (Ref) & 342 & - & - & - \\
\hline $20-24$ & 1027 & 1.069 & $0.645-1.234$ & 0.000 \\
\hline $25-29$ & 1258 & 1.996 & $0.756-2.089$ & 0.000 \\
\hline $30-34$ & 1313 & 2.007 & $1.001-2.895$ & 0.000 \\
\hline $35-39$ & 1219 & 2.568 & $1.234-3.009$ & 0.000 \\
\hline $40-44$ & 937 & 3.001 & $2.334-3.345$ & 0.002 \\
\hline $45-49$ & 627 & 3.045 & $2.675-4.003$ & 0.000 \\
\hline \multicolumn{5}{|l|}{ Residence } \\
\hline Urban & 2662 & 1.859 & $1.731-1.997$ & 0.000 \\
\hline Rural (Ref) & 4061 & - & - & - \\
\hline \multicolumn{5}{|l|}{ Standard of living Index } \\
\hline Low (Ref) & 1338 & - & - & - \\
\hline Medium & 2333 & 1.918 & $1.770-2.070$ & 0.000 \\
\hline High & 3052 & 2.465 & $1.845-2.999$ & 0.001 \\
\hline \multicolumn{5}{|l|}{ Religion } \\
\hline Hindu (Ref) & 5461 & - & - & - \\
\hline Non-Hindu & 1262 & 1.698 & $0.567-3.008$ & 0.000 \\
\hline \multicolumn{5}{|l|}{ Respondent's Education } \\
\hline Illiterate (Ref) & 3840 & - & - & - \\
\hline Primary & 758 & 1.268 & $1.136-1.416$ & 0.000 \\
\hline Secondary & 1513 & 1.526 & $1.400-1.663$ & 0.000 \\
\hline Higher & 612 & 2.504 & $2.183-2.872$ & 0.000 \\
\hline \multicolumn{5}{|l|}{ Partner's Education } \\
\hline Illiterate( Ref) & 1752 & - & - & - \\
\hline Primary & 776 & 1.084 & $0.968-1.213$ & 0.163 \\
\hline Secondary & 3122 & 1.363 & $1.259-1.475$ & 0.000 \\
\hline Higher & 1073 & 2.139 & $1.911-2.393$ & 0.000 \\
\hline \multicolumn{5}{|l|}{ Respondent's Occupation } \\
\hline Service class & 370 & 1.715 & $1.276-2.305$ & 0.000 \\
\hline Physical worker & 1919 & 0.864 & $0.901-1.310$ & 0.384 \\
\hline House-wives (Ref) & 4434 & - & - & - \\
\hline \multicolumn{5}{|l|}{ Currently Breast feeding } \\
\hline Yes (Ref) & 2190 & - & - & - \\
\hline No & 4533 & 1.234 & $0.456-2.008$ & 0.000 \\
\hline \multicolumn{5}{|l|}{ Anaemia } \\
\hline Yes (Ref) & 3305 & - & - & - \\
\hline No & 3418 & 1.861 & $1.002-2.056$ & 0.000 \\
\hline
\end{tabular}




\section{Reference}

[1] Arnold, F., Choe, M.K. and Roy, T.K. (1996). Son preference, the family-building process and child mortality in India. Population Studies, 52, 301-315.

[2] Bang R.A., Bang, A.T., Baitule, Y., Choudhary, S., Sarmukkadam, S. and Tale, O. (1989). High prevalence of gynecological diseases in rural Indian women. Lancet, 14(8629), 85-88.

[3] Basu, A.M. (1995). Women's roles and the gender gap in health and survival. In: Women's health in India: risk and vulnerability, edited by Monica Das Gupta, Lincoln C. Chen, T.N. Krishnan. New Delhi, India, Oxford India Paperbacks, 153-174.

[4] Bhatia, J.C. and Cleland, J. (1995). Self-reported symptoms of gynecological morbidity and their treatment in South India. Stud Family Planning, 26(4), 203- 216

[5] Brabin, L., Nicholas, S., Gogate, K. and Karande, A. (1998). High prevalence of anemia among women in Mumbai. India Food Nutr. Bull., 19(3), 205-222.

[6] Cawley, J. (2016). The impact of health insurance on preventive care and health behaviours: Evidence from the 2014 ACA Medicade Expainsons. National Bureau of Economic Research, Working Paper No. 22265. http://www.nber.org/papers/w22265

[7] Cawley, J., Moran, J. and Simon, K. (2008). The impact of income on the weight of elderly Americans. National Bureau of Economic Research, Working Paper No. 14104.

[8] Cheng, P.P., Jiao, X.Y., Wang, X.H., Lin, J.H. and Cai, Y.M. (2011). Hepcidin expression in anemia of chronic disease and concomitant iron-deficiency anemia. ClinExp Med, 11(1), $33-42$.

[9] Felix, F., John, T., Wulu, J.T and Karan P. Singh (2004). On the Generalized Poisson Regression Model with an Application to Accident Data. Journal of Data Science, 2, 287295

[10] Galloway, R. and McGuire, J. (1994). Determinants of compliance with iron supplementation: supplies, side effect, or psychology. Soc Sci Med, 39, 381-390.

[11] International Institute for Population Sciences \& ORC Macro National Family Health Survey India 2005/06.IIPS, Mumbai, India.

[12] International Institute for Population Sciences (IIPS), World Health Organization (WHO), and World Health Organization (WHO)-India-WR Office. 2006. Health System Performance Assessment: World Health Survey 2003 India. Mumbai: IIPS.

[13] Kishor, S. (1998). Gender differentials in child mortality: a review of the evidence. In: Women's health in India: risk and vulnerability, edited by Monica Das Gupta, Lincoln C. Chen, T.N. Krishnan. New Delhi, India, Oxford India Paperbacks, 19-54.

[14] Koblinsky, M.A. (1995). Beyond maternal mortality-magnitude, interrelationship and consequences of women's health, pregnancy related complications and nutritional status on pregnancy out-comes. Int J Gynecol Obstetr ,48, 21-32.

[15] Miller, B.D. (1983). The endangered sex: neglect of female children in rural north India. The Journal of developing areas, 17(4), 521-523. 
Regression

[16] Schmeiser, M. (2008). Expanding wallets and waistlines: The impact of family income on the BMI of women and men eligible for the Earned Income Tax Credit. Unpublished manuscript, Department of Consumer Science, University of Wisconsin at Madison.

[17] Sobal, J. and Stunkard, A.J. (1989). Socioeconomic status and obesity: a review of the literature. Psychological Bulletin 105(2), 260-275.

[18] Vijayaraghavan, K., Brahmam, G.N., Nair, K.M., Akbar, D. and Rao N.P. (1990). Evaluation of National Nutritional Anemia Prophylaxis Program. Ind J Pediatr, 57(2), 183 190.

[19] World Health Organization (2003). Iron deficiency anemia: assessment, prevention, and control. A guide for program managers. Geneva: World Health Organization.

\author{
Brijesh P. Singh \\ Faculty of Commerce \& DST-CIMS, \\ Banaras Hindu University, Varanasi \\ Sonam Maheshwari \\ Department of Community Medicine \\ SRMS-IMS, Bareilly \\ Puneet Kumar Gupta \\ Department of Statistics \\ University of Allahabad, Allahabad
}

